\title{
Linfohistiocitosis hemofagocítica como presentación de Linfoma de células-T
}

\author{
Hemophagocytic lymphohistiocytosis as a presentation of T-cell lymphoma \\ Linfohistiocitose hemofagocítica como uma apresentação de linfoma de células $T$
}

\author{
Silvina Ayelén Casares Diaz ${ }^{1}$, Enzo Marcelo Amelia², Martín Milanesio ${ }^{3}$, Luciana Guanchiale ${ }^{3,4}$, Juan Manuel Alomar ${ }^{1,4}$, \\ Emanuel José Saad ${ }^{1,4,5}$.
}

La linfohistiocitosis hemofagocítica es un síndrome que se produce por un aumento en la actividad de diferentes células del organismo conduciendo a un estado hipe rinflamatorio. Se puede presentar habitualmente como un síndrome febril asociado a disminución de los glóbulos blancos, glóbulos rojos y/o plaquetas en la sangre periférica, aumento de tamaño del bazo e hígado, $y$ alteraciones analíticas como aumento de ferritina y triacilglicéridos y disminución de fibrinógeno. Su forma de presentación asimismo puede ser secundaria a complicaciones de este proceso, como por ejemplo produciéndose infecciones asociadas. Posee un pobre pronóstico, por lo cual su sospecha clínica debe ser precoz para instaurar tratamientos pertinentes.

\section{Conceptos clave}

Que se sabe sobre el tema:

-La linfohistiocitosis hemofagocítica es un síndrome hiperinflamatorio que puede ser primario o secundario a enfermedades infecciosas, autoinmunes o neoplasias.

-Tiene una elevada mortalidad, por lo que el diagnóstico temprano con inicio precoz de terapia adecuada son claves en el manejo de estos pacientes.

-El linfoma de células T es un linfoma no Hodgkin que en escasas ocasiones puede manifestarse de manera inicial mediante una linfohistiocitosis hemofagocítica.

\section{Que aporta este trabajo:}

-Presentamos el caso de una paciente femenina con linfohistiocitosis hemofagocítica como manifestación inicial de un linfoma de células $T$.

-La forma de presentación fue atípica manifestando no sólo características propias de la linfohistiocitosis hemofagocítica, sino también manifestaciones infecciosas virales, fúngicas y bacterianas secundarias a la inmunodepresión provocada.

1- Servicio de Clínica Médica de Hospital Privado Universitario de Córdoba,

2- Servicio de Anestesiología de Hospital Privado Universitario de Córdoba.

3- Servicio de Hematología y Oncología de Hospital Privado Universitario de Córdoba.

4- Instituto Universitario de Ciencias Biomédicas de Córdoba.

5- E-mail de contacto: emanuelsaad@hotmail.com

Recibido: 2020-09-20 Aceptado: 2021-06-12

DOI: https://doi.org/10.31053/1853.0605.v78.n3.30373

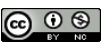

https://creativecommons.org/licenses/by-nc/4.0/

(c) Universidad Nacional de Córdoba

\section{Resumen:}

Introducción: La linfohistiocitosis hemofagocítica (LHH) es un síndrome hiperinflamatorio severo causado por activación aberrante de macrófagos y células $T$ citotóxicas que se manifiesta clínicamente como un cuadro febril asociado a citopenias, hiperferritininemia y esplenomegalia. Ante su diagnóstico en adultos se deben buscar causas secundarias como neoplasias hematológicas. El inicio precoz del tratamiento es importante debido a su elevada mortalidad.

Métodos: Se presenta el caso de una paciente de 53 años de edad que se le realizó diagnóstico de LHH asociada a Linfoma de células T. La forma de presentación de dicho cuadro fue de manera abrupta con varios síntomas y signos de dicho síndrome, además de múltiples complicaciones infecciosas asociadas.

Resultados: Ante el diagnóstico inicial de LHH se inició tratamiento con etopósido y dexametasona, y al obtener el diagnóstico de Linfoma de células $\mathrm{T}$, se instauró tratamiento quimioterápico con esquema de CHOEP, con evolución inicial favorable. Conclusiones: La LHH es una entidad poco frecuente asociada a una elevada mortalidad, por lo que su índice de sospecha debe de ser elevado ante un cuadro clínico y analítico compatible. Sin embargo, su diagnóstico constituye un gran desafío clínico, pudiendo presentarse en algunas ocasiones superpuesto a cuadros infecciosos múltiples. El inicio precoz del tratamiento es importante debido a su alta mortalidad.

Palabras clave: linfohistiocitosis hemofagocítica; linfoma; huésped inmunocomprometido; linfadenopatía.

\section{Abstract:}

Introduction: Hemophagocytic lymphohistiocytosis $(\mathrm{HHL})$, a severe hyperinflammatory syndrome caused by aberrant activation of macrophages and cytotoxic $\mathrm{T}$ cells, is clinically manifested as a febrile onset along with cytopenias, high ferritin serum level and splenomegaly. In adult patients, secondary causes of it should be looked for, such as autoimmune and infectious diseases and neoplasms. Prompt initiation of treatment is important due to the high mortality of this syndrome.

Methods: A case of a 53-year-old patient diagnosed with HHL associated with T-cell lymphoma is presented as a sudden onset of several symptoms and signs of $\mathrm{HHL}$, along with infectious complications.

Results: Once the diagnosis of $\mathrm{HHL}$ was made, treatment with Etoposide and Dexamethasone was started, and with the diagnosis of T-cell lymphoma established, chemotherapy treatment with a CHOEP scheme was started, with a favorable initial evolution.

Main conclusion: As HHL is a rare entity associated with high mortality, initial suspicion must be high when facing an acute onset of fever, cytopenia and splenomegaly. Nonetheless, diagnosing $\mathrm{HHL}$ is challenging and often appear superimposed on multiple infectious diseases. The early initiation of treatmbent is important given the high mortality of this pathology

Keywords: lymphohistiocytosis, hemophagocytic; lymphoma; immunocompromised host; lymphadenopathy.

\section{Resumo:}

Introdução: A linfo-histiocitose hemofagocítica (HHL) é uma síndrome hiperinflamatória grave causada pela ativação aberrante de macrófagos e células $T$ citotóxicas que se manifesta clinicamente como quadro febril associado a citopenias, ferritina elevada e esplenomegalia. Antes de seu diagnóstico em adultas, as causas secundárias, como as neoplasias hematológicas, devem ser investigadas. O início precoce do tratamento é importante devido à sua alta mortalidade.

Métodos: Apresentamos o caso de um paciente de 53 anos de idade com diagnóstico de HHL associado a linfoma de células T. A apresentação do quadro foi abrupta com diversos sintomas e sinais da referido síndrome, além de múltiplas complicações infecciosas associadas.

Resultados: Antes do diagnóstico inicial de HHL, iniciou-se o tratamento com etoposídeo e dexametasona, e quando o diagnóstico de linfoma de células T foi obtido, iniciou-se o tratamento quimioterápico com esquema CHOEP, com evolução inicial favorável.

Conclusões: $\mathrm{O}$ HHL é uma entidade rara associada a alta mortalidade, portanto seu índice de suspeita deve ser alto quando se depara com um quadro clínico e analítico compatível. No entanto, seu diagnóstico constitui um grande desafio clínico, podendo, às vezes, aparecer sobreposto a múltiplos quadros infecciosos. O início precoce do tratamento é importante devido à alta mortalidade dessa patologia.

Palavras-chave: linfo-histiocitose hemofagocítica; linfoma; hospedeiro imunocomprometido; linfadenopatia. 


\section{INTRODUCCIÓN}

La linfohistiocitosis hemofagocítica (LHH) constituye un infrecuente y severo síndrome hiperinflamatorio producido por la activación aberrante de macrófagos y células-T citotóxicas ${ }^{(1,2,3)}$. Existe una forma primaria que es casi exclusiva de la población pediátrica y una secundaria que es más frecuente en la población adulta. Esta última puede ser desencadenada por enfermedades infecciosas, autoinmunes o neoplásicas. Recientemente se ha propuesto utilizar la denominación de "síndrome de LHH" para aquellos casos en que los pacientes presentan suficientes características diagnósticas de LHH, mientras que se considera "enfermedad de LHH" sólo a un subconjunto de éstos en los cuales la desregulación inmune distintiva es el problema central, como ocurre en pacientes con LHH hereditaria $^{(4)}$.

Su diagnóstico plantea un gran desafío debido a que sus manifestaciones suelen simular otros cuadros como la sepsis y el daño multiorgánico ${ }^{(2)}$. Es importante la sospecha diagnóstica temprana, debido a que el retardo en el inicio del tratamiento se ha asociado a un aumento de la mortalidad ${ }^{(1)}$.

A continuación, reportamos el caso de un paciente con linfoma de células-T que se ha presentado como LHH.

\section{CASOS CLÍNICOS}

Mujer de 53 años que dos días después de haber arribado a Argentina desde Estados Unidos de América comienza con síndrome febril, constatándose temperatura de $39^{\circ} \mathrm{C}$, asociado a dolor abdominal difuso y deposiciones líquidas sin otros productos patológicos. Negaba otros síntomas asociados como pérdida de peso corporal significativa, disnea o cuadros infecciosos en los meses previos. La paciente tenía como antecedentes patológicos obesidad e hipotiroidismo. No tenía antecedentes quirúrgicos previos ni hábitos tóxicos de relevancia. Al examen físico presentaba molestias a la palpación profunda en hemiabdomen superior, sin otra particularidad. Una ecografía abdominal no reveló alteraciones significativas y en el laboratorio se destacaba sólo discreta leucocitosis de $14000 \mathrm{cel} / \mu \mathrm{L}$, con valores de hemoglobina, plaquetas, creatinina y transaminasas dentro de límites normales. Se interpretó cuadro clínico como gastroenteritis y se indicó tratamiento sintomático.

Debido a persistencia de la sintomatología volvió a consultar a las 96 horas. Al examen físico estaba febril $\left(39,5^{\circ} \mathrm{C}\right)$, taquicárdica y taquipneica. Presentaba muguet oral y adenopatías cervicales bilaterales y axilar izquierda. En pared torácica anterior se observaron lesiones vesiculares sugestivas de virus herpes simplex. En el laboratorio se objetivó leucocitosis con bicitopenia (anemia y plaquetopenia), hiperferritinemia, hipertrigliceridemia, hiperbilirrubinemia a predominio directo junto con elevación de creatinina, enzimas hepáticas, lactato deshidrogenasa humana $(\mathrm{LDH})$, proteína-C reactiva(PCR), eritrosedimentación y de procalcitonina (Tabla 1). Serología viral $(\mathrm{VIH}$, virus hepatitis $\mathrm{B}$ y $\mathrm{C}$, Citomegalovirus, Virus Epstein-Barr y Dengue) y anticuerpos antinucleares fueron negativos, y los valores de complemento $\mathrm{C} 3$ y C4 fueron normales. En tomografía toraco-abdominal se observó esplenomegalia de $17 \mathrm{~cm}$ con lesiones infiltrativas nodulares y numerosas adenomegalias cervicales, supraclaviculares, axilares, mediastinales y retroperitoneales. Debido a la presencia de fiebre, organomegalia, adenomegalia, anemia, plaquetopenia, hiperferritinemia, hipertrigliceridemia y descenso de fibrinógeno, se sospechó que podía tratarse de un síndrome de LHH. Ante la existencia de posibles desencadenantes se inició tratamiento empírico antimicrobiano con ceftriaxona y doxiciclina (por sospecha de patología bacteriana asociada, teniendo valores de procalcitonina y PCR elevados), antiviral con aciclovir (por lesiones sugestivas de herpes) y nistatina oral (por muguet) y se realizó biopsia de ganglio cervical en búsqueda de diagnóstico de etiología neoplásica o infecciosa. En hemocultivos se realizó aislamiento de Candida albicans, por lo cual se inició tratamiento antifúngico endovenoso con fluconazol. 
Tabla N¹: Evolución de parámetros de laboratorio

\begin{tabular}{|c|c|c|c|}
\hline Parámetro de laboratorio & $\begin{array}{c}\text { Día de ingreso } \\
\text { hospitalario }\end{array}$ & $\begin{array}{c}\text { Día } 6 \text { (Inicio tratamiento } \\
\text { LHH) }\end{array}$ & $\begin{array}{c}\text { Día } 26 \text { (día de } \\
\text { alta hospitalaria) }\end{array}$ \\
\hline Leucocitos (cel/ $/ \mu \mathrm{L})$ & 16000 & 30000 & 11000 \\
\hline Neutrófilos en banda (cel/ $\mu \mathrm{L})$ & 800 & 300 & 0 \\
\hline Neutrófilos segmentados $(\mathrm{cel} / \mu \mathrm{L})$ & 9600 & 12900 & 7000 \\
\hline 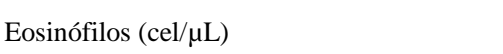 & 0 & 0 & 0 \\
\hline Basófilos (cel/ $/ \mu \mathrm{L})$ & 0 & 0 & 0 \\
\hline Linfocitos (cel/ $/ \mu \mathrm{L})$ & 2240 & 1500 & 2500 \\
\hline Monocitos (cel/ $/ \mu \mathrm{L})$ & 3360 & 1800 & 1500 \\
\hline Hemoglobina $(\mathrm{g} / \mathrm{dL})$ & 10 & 7.7 & 8,4 \\
\hline VCM (fL) & 82 & 84 & 88 \\
\hline $\mathrm{HCM}(\mathrm{pg})$ & 28 & 28 & 28 \\
\hline CHCM (gr/dL) & 35 & 34,4 & 32,7 \\
\hline RDW (\%) & 14,9 & 16,3 & 16,3 \\
\hline Plaquetas (plaquetas/ $\mu \mathrm{L}$ ) & 22000 & 12000 & 150000 \\
\hline Eritrosedimentación (mm en $1^{\circ}$ hora) & 46 & - & - \\
\hline Proteína $\mathrm{C}$ reactiva (mg/dL) & 24,06 & 3,88 & 7,95 \\
\hline Procalcitonina (ng/mL) & 95 & & - \\
\hline Ferritina (ng/mL) & 2928 & 14040 & 2646 \\
\hline Triacilgliceridemia (mg/dL) & 329 & 350 & 190 \\
\hline Fibrinogenemia (mg/dL) & 516 & 140 & 150 \\
\hline Creatinina $(\mathrm{mg} / \mathrm{dL})$ & 1,84 & 1,6 & 0,42 \\
\hline $\mathrm{AST}(\mathrm{U} / \mathrm{L})$ & 118 & 144 & 98 \\
\hline $\mathrm{ALT}(\mathrm{U} / \mathrm{L})$ & 91 & 120 & 100 \\
\hline FAL (U/L) & 244 & 780 & 600 \\
\hline GGT (UI/L) & 323 & 1560 & 1000 \\
\hline LDH (U/L) & 539 & 529 & 400 \\
\hline Bilirrubina total/Bilirrubina directa (mg/dl) & $1.57 / 1,42$ & $6.38 / 6,06$ & $2.61 / 1,91$ \\
\hline TP (segundos) & 1,4 & 6 & 1,9 \\
\hline $\begin{array}{l}\text { TTPa (Segundos) } \\
\text { LHH: Lifohistiocitosis hemofagocítica; Cel: células.; }\end{array}$ & $\frac{47453}{\text { men corpuscular me }}$ & $\frac{45627}{\text { M: hemoglobina corpuscular me }}$ & $\begin{array}{c}44501 \\
\text { CHCM: concentración }\end{array}$ \\
\hline
\end{tabular}

La paciente presentó posteriormente descenso paulatino de leucocitosis, PCR y procalcitonina, pero profundizó cuadro de anemia, plaquetopenia (con necesidad transfusional) e hipofibrinogenemia, con elevación de ferritina (llegando a $18960 \mathrm{ng} / \mathrm{mL}$ ), dímero $\mathrm{D}$, bilirrubina total (llegando a $8,17 \mathrm{mg} / \mathrm{dL}$ ) y transaminasas. En la Tabla 1 se puede observar la evolución de los valores de laboratorio a lo largo de la internación. Ante dicho escenario al sexto día de ingreso hospitalario se decidió iniciar tratamiento del síndrome LHH inicialmente con dexametasona $20 \mathrm{mg} / \mathrm{día}$ y posteriormente se agregó etopósido $100 \mathrm{mg} / \mathrm{m}^{2}$, recibiendo un total de tres dosis, tras la cual se obtuvo el resultado de anatomía patológica de la biopsia ganglionar. En la misma se observó distorsión de arquitectura ganglionar reemplazada por proliferación de células linfoides con inmunomarcación positiva para estirpe T CD7 de manera difusa y para CD30 con patrón dot-like, CD3 escasas células positivas, además de observarse positivo CD4 y negativo CD8, CD56 y BCL-6, teniendo Ki67 de 80\%. Estos signos eran compatibles con Linfoma $T$ periférico no especificado (NOS), no anaplásico CD30+. En médula ósea se observó sólo hiperplasia mieloide sin infiltración por proceso linfoproliferativo. De acuerdo a los hallazgos observados, el proceso linfoproliferativo se encontraba en un estadío por Ann Arbor IIIB, con riesgo medio-alto según índice pronóstico internacional (IPI) y grupo 3 según índice pronóstico de linfomas $\mathrm{T}(\mathrm{PIT})$. Ante dicho diagnóstico el plan terapéutico fue de iniciar tratamiento quimioterápico con ciclofosfamida, doxorrubicina, vincristina, etopósido y 
prednisona(CHOEP) realizando el primer ciclo en nuestro hospital y posteriormente realizó 2 ciclos más en otra institución sin respuesta, por lo que se encuentra en planes de trasplante alogénico de progenitores hematopoyéticos.

\section{DISCUSIÓN}

La LHH es un síndrome hiperinflamatorio que conduce a un severo daño tisular secundario a la infiltración tisular de los propios macrófagos y células linfocitarias, así como también por las citoquinas proinflamatorias secretadas ${ }^{(2,5)}$. Ante su sospecha diagnóstica se debe de iniciar la búsqueda de posibles desencadenantes, ya que su tratamiento será clave en el pronóstico de la enfermedad. Si bien las infecciones representan una de las causas más frecuentes, cerca del $40 \%$ de los casos de $\mathrm{LHH}$ en adultos están asociados a patologías neoplásicas, pudiendo ocurrir al inicio de la enfermedad o durante su tratamiento ${ }^{(6)}$. Sin embargo, la incidencia de $\mathrm{LHH}$ en pacientes con enfermedad oncohematológica es cerca del $1 \%$. Las principales neoplasias asociadas son de células-T o natural killer (NK) (35\%) y linfomas de células B $(32 \%)$, seguido de leucemias $(6 \%)$ y linfoma Hodgkin $(6 \%)$ y tumores sólidos $(3 \%)^{(2)}$.

Uno de los criterios diagnósticos más empleados en la actualidad son los LHH-2004 revisados, que requieren la presencia de 5 de los siguientes 8 datos clínicos/analíticos: a) fiebre $\geq 38.5^{\circ} \mathrm{C}$; b) esplenomegalia, c) citopenias en sangre periférica con al menos dos de las siguientes: hemoglobina $<9 \mathrm{~g} / \mathrm{dL}$, plaquetas $<100000 / \mathrm{uL}$, conteo de neutrófilos<1000/uL; d) hipertrigliceridemia en ayunas(>265 $\mathrm{mg} / \mathrm{dL}) \quad \mathrm{y} / \mathrm{o} \quad$ hipofibrinogenemia (fibrinógeno $<150 \mathrm{mg} / \mathrm{dL}$ ); e) hemofagocitosis en la médula ósea, bazo, ganglios linfáticos o hígado; f) actividad de células NK baja o ausente; g) ferritina $>500 \mathrm{ng} / \mathrm{mL}$, h) elevación de CD25 soluble $>240 \mathrm{U} / \mathrm{mL}^{(7)}$. Sin embargo, estos criterios sólo han sido validados en la población pediátrica y no en la adulta. Por ello, se debe tener en cuenta que muchos pacientes presentan sólo tres o cuatro de los criterios pero tienen evidencia clínica de LHH y requieren tratamiento específico ${ }^{(3)}$. A pesar de no contar con el dosaje de CD25 soluble ni con la actividad de células NK, la paciente presentada tenía 5 criterios (fiebre, esplenomegalia, anemia y trombocitopenia, hipertrigliceridemia/hipofibrinogenemia e hiperferritinemia) permitiendo aproximarse al diagnóstico. Si bien la demostración de hemofagocitosis es bastante característica de esta entidad, no es patognomónica y no es un criterio excluyente para su diagnóstico. Incluso puede observarse hemofagocitosis en cuadros diferentes a $\mathrm{LHH}$, como infecciones, posterior a transfusiones sanguíneas e incluso en sujetos sanos ${ }^{(8,9)}$. Si bien la neutropenia es una de las citopenias más frecuentes en pacientes con LHH, creemos que en nuestro caso pudo estar enmascarada por la presencia de infección concomitante.

Por otro lado, se ha propuesto recientemente que el H-Score se ajustaría más a pacientes adultos ${ }^{(10)}$. Este pondera 9 variables, de las cuales 3 son clínicas (inmunosupresión, fiebre y organomegalia), 5 analíticas (triglicéridos, ferritina, aspartato aminotransferasa, fibrinógeno y citopenias) y 1 citológica (hemofagocitosis en aspirado de médula ósea). Este score tiene una sensibilidad del $90 \%$ y especificidad del $79 \%$ en adultos ${ }^{(11)}$. En nuestro caso la paciente poseía un $\mathrm{H}$-Score con 239 puntos, que indicaría una probabilidad de $98-99 \%$ de $\mathrm{LHH}$. Si bien varios de los hallazgos clínicos y analíticos que presentó nuestra paciente podían explicarse también por el linfoma, en un análisis retrospectivo de pacientes con linfoma de células- $T$ se ha observado que tanto la fiebre, bicitopenia, hepatoesplenomegalia, hiperferritinemia, aumento de $\mathrm{LDH}$, hipertrigliceridemia, hipofibrinogenemia y alteración en hepatograma se presentaron con mayor frecuencia en los casos asociados a $\mathrm{LHH}$ respecto a aquellos sin asociación al mismo ${ }^{(12)}$. De esta forma se propone que en pacientes con diagnóstico linfoma $T$, la presencia de varios de dichos hallazgos deben orientar a la búsqueda de un síndrome de LHH sobreagregado. Nuestro paciente, a excepción de hepatomegalia, presentó todas las alteraciones clínicas y de laboratorio citadas anteriormente, lo que sugería aún más al diagnóstico de síndrome de $\mathrm{LHH}$ asociado. Como se puede observar, ningún elemento clínico o de laboratorio por sí solo tiene elevada sensibilidad y especificidad para el diagnóstico de $\mathrm{LHH}^{(2,4)}$. La LHH se asocia a una elevada mortalidad en los adultos, reportada entre un $50-74 \%$. Las principales causas de mortalidad son complicaciones hemorrágicas, infecciones o consecuencias asociadas a las enfermedades de base ${ }^{(5,13,14)}$. En el caso de estar asociado a linfoma, el pronóstico es aún más sombrío cuando se trata de células- $\mathrm{T}^{(4)}$. Por dicho motivo, en estos casos resulta de gran importancia realizar un manejo terapéutico intensivo precoz ${ }^{(15)}$. Una revisión de consenso reciente sugirió un enfoque de dos pasos en los casos de LHH asociada a malignidad en adultos. Primero, se ataca la tormenta de citocinas y la proliferación de células $T$ (inicialmente con esteroides, etopósido e inmunoglobulinas) y en el segundo paso se instaura la terapia dirigida a la neoplasia maligna subyacente ${ }^{(15)}$. Tras el tratamiento inicial, los pacientes con recidiva, persistencia 0 malignidad hematológica sin respuesta a quimioterapia, como ocurrió en nuestro paciente, deben evaluarse para recibir trasplante alogénico de progenitores hematopoyéticos ${ }^{(5)}$.

\section{CONCLUSIONES}

La LHH es una entidad poco frecuente asociada a una elevada mortalidad, por lo que su índice de sospecha debe de ser elevado ante un cuadro clínico y analítico compatible. Sin embargo, su diagnóstico constituye un gran desafío clínico, pudiendo presentarse en algunas ocasiones superpuesto a cuadros infecciosos múltiples.

\section{Limitaciones de responsabilidad:}

Los autores del presente artículo se desempeñan en la institución Hospital Privado Universitario de Córdoba. No se poseen fuentes de financiación externas.

\section{Fuentes de apoyo:}

Se declara que los autores no presentaron fuentes de apoyo como subvenciones, equipos, medicamentos y/o cualquier otro apoyo que haya contribuido a la investigación o escritura del presente manuscrito.

\section{Originalidad:}

Este manuscrito no ha sido publicado, parcial o totalmente, en ninguna otra revista.

\section{Cesión de derechos:}

Manifestamos nuestra conformidad de otorgar los derechos de autor a la Universidad Nacional de Córdoba para publicar en la Revista de la Facultad de Ciencias Médicas de la Universidad Nacional de Córdoba si se concreta la publicación del manuscrito en vuestra revista.

Conflicto de interés:

Los autores declaran no poseer conflictos de interés.

\section{BIBLIOGRAFÍA}

1. Lee DE, Martinez-Escala ME, Serrano LM, Zhou XA, Kaplan JB, Pro B, Choi J, Guitart J. Hemophagocytic Lymphohistiocytosis in Cutaneous T-Cell Lymphoma. JAMA Dermatol. 2018 Jul 1;154(7):828-831. doi: 10.1001/jamadermatol.2018.1264.

2. La Rosée P, Horne A, Hines $M$, von Bahr Greenwood $T$, Machowicz R, Berliner N, Birndt S, Gil-Herrera J, Girschikofsky M, Jordan MB, Kumar A, van Laar JAM, Lachmann G, Nichols KE, Ramanan AV, Wang $Y$, Wang $Z$, Janka G, Henter Jl. Recommendations for the management of hemophagocytic lymphohistiocytosis in adults. Blood. 2019 Jun 6;133(23):2465-2477. doi: 10.1182/blood.2018894618.

3. Otrock ZK, Daver N, Kantarjian HM, Eby CS. Diagnostic Challenges of Hemophagocytic Lymphohistiocytosis. Clin Lymphoma Myeloma Leuk. 2017 Jul;17S:S105-S110. doi: 10.1016/j.clml.2017.02.017. 


\section{LINFOHISTIOCITOSIS HEMOFAGOCÍTICA}

4. Jordan MB, Allen CE, Greenberg J, Henry M, Hermiston ML, Kumar A, Hines M, Eckstein O, Ladisch S, Nichols KE, RodriguezGalindo C, Wistinghausen B, McClain KL. Challenges in the diagnosis of hemophagocytic lymphohistiocytosis: Recommendations from the North American Consortium for Histiocytosis (NACHO). Pediatr Blood Cancer. 2019 Nov;66(11):e27929. doi: 10.1002/pbc.27929.

5. Martín-Gómez MA, Caba-Molina M, Viciana-Martínez-Lage MJ, Ortega-Salas R, Sánchez-Crespo A, Soriano-Pérez MJ, GómezMorales M. Glomerulonefritis colapsante con marcadores de desdiferenciación podocitaria en síndrome hemofagocítico secundario a linfoma hepatoesplénico de células $T$. Biomedica. 2018;38(4):456-462. doi: 10.7705/biomedica.v38i4.3893

6. Bhatt NS, Oshrine B, An Talano J. Hemophagocytic lymphohistiocytosis in adults. Leuk Lymphoma. 2019 Jan;60(1):1928. doi: 10.1080/10428194.2018.1482543.

7. Henter Jl, Horne A, Aricó $M$, Egeler RM, Filipovich $A H$, Imashuku S, Ladisch S, McClain K, Webb D, Winiarski J, Janka G. HLH-2004: Diagnostic and therapeutic guidelines for hemophagocytic lymphohistiocytosis. Pediatr Blood Cancer. 2007 Feb;48(2):124-31. doi: 10.1002/pbc.21039

8. McGinnis E, Medvedev N, Richards MJ, Chen LYC, Wong MP. Post-Transfusion Hemophagocytosis Without Hemophagocytic Lymphohistiocytosis. Mayo Clin Proc Innov Qual Outcomes. 2019 Oct 16;3(4):517-522. doi: 10.1016/j.mayocpiqo.2019.07.001.

9. Ho C, Yao X, Tian L, Li FY, Podoltsev N, XU ML. Marrow assessment for hemophagocytic lymphohistiocytosis demonstrates poor correlation with disease probability. Am J Clin Pathol. 2014 Jan;141(1):62-71. doi: 10.1309/AJCPMD5TJEFOOVBW.

10. Fardet L, Galicier L, Lambotte O, Marzac C, Aumont C, Chahwan D, Coppo P, Hejblum G. Development and validation of the HScore, a score for the diagnosis of reactive hemophagocytic syndrome. Arthritis Rheumatol. 2014 Sep;66(9):2613-20. doi: 10.1002/art.38690.

11. Al-Samkari H, Berliner N. Hemophagocytic Lymphohistiocytosis. Annu Rev Pathol. 2018 Jan 24;13:27-49. doi: 10.1146/annurevpathol-020117-043625.

12. Tong H, Ren Y, Liu H, Xiao F, Mai W, Meng H, Qian W, Huang J, Mao L, Tong Y, Wang L, Qian J, Jin J. Clinical characteristics of T-cell lymphoma associated with hemophagocytic syndrome: comparison of T-cell lymphoma with and without hemophagocytic syndrome. Leuk Lymphoma. 2008 Jan;49(1):81-7. doi: 10.1080/10428190701713630.

13. Morimoto A, Nakazawa $Y$, Ishii E. Hemophagocytic lymphohistiocytosis: Pathogenesis, diagnosis, and management. Pediatr Int. 2016 Sep;58(9):817-25. doi: 10.1111/ped. 13064.

14. Campo M, Berliner N. Hemophagocytic Lymphohistiocytosis in Adults. Hematol Oncol Clin North Am. 2015 Oct;29(5):915-25. doi: 10.1016/j.hoc.2015.06.009.

15. Pasvolsky O, Zoref-Lorenz A, Abadi U, Geiger KR, Hayman L, Vaxman I, Raanani $P$, Leader A. Hemophagocytic lymphohistiocytosis as a harbinger of aggressive lymphoma: a case series. Int J Hematol. 2019 May;109(5):553-562. doi: 10.1007/s12185-019-02623-z. 